\title{
Among the trees: iterating geneses of forms, in art and in nature.
}

\section{Entre les arbres : itérer la genèse des formes, en art et dans la nature.}

\author{
Giuseppe Longo ${ }^{1}$, Sara Longo ${ }^{2}$ \\ ${ }^{1}$ CNRS, Ecole Normale Sup., Paris, \\ https://www.di.ens.fr/users/longo/ \\ ${ }^{2}$ Paris 1, Panthéon-Sorbonne, \\ https://hicsa. univ-paris 1. fr/page. php?r=23\&id=216\&lang=fr \\ Publié le 6 février 2020 DOI : 10.21494/ISTE.OP.2020.0473
}

\begin{abstract}
In many dynamics of nature we may witness the iteration of a morphogenetic process. Mandelbrot fractals allow us to give an elegant mathematical representation of those engendered by physical processes and provide new tools for an insight into some dynamics in Quantum Physics. The art photography book, reviewed here, proposes an original perspective of the forms and shadows of trees' branches and leaves. It stresses the nonalgorithmic diversity of biological morphogenesis, by adding also the artist's interpretation by double exposures and filtering techniques. Analogies with two modern figurative painters will be hinted as well as the distortion of knowledge and economic action induced by fast-trading and the search of fractal regularities in the stock-market.

KEYWORDS. Mathematical fractals, art photography, shadows in painting, biological morphogenesis, physical iteration, economic dynamics, Benoît Mandelbrot, René Magritte, Simon Hantaï, Luca Caciagli.
\end{abstract}

A recent collection of pictures by the art photograph Luca Caciagli is introduced by the author as follows:

The "Komorebi" project is inspired by fractal geometry.

"Komorebi" is an untranslatable Japanese word, which eloquently captures the effect of sunlight streaming through the leaves of the trees.

The term "fractal" was coined by the mathematician Benoît Mandelbrot, to describe those irregular forms of nature, such as a cloud, a coast, a tree. Extending any part of a fractal gives a figure similar to the original. I believe that photography can make us imagine more complete relationships between man and nature, based on concepts of unity, interdependence and equality, inherent in the idea of fractals. In Komorebi, the affinities in the fractal geometric structure are reinterpreted with the double exposure trying to distance the viewer from the classic identification of a subject present in nature.

Instagram: @lu.caciagli Web page: www.caciagliluca.com
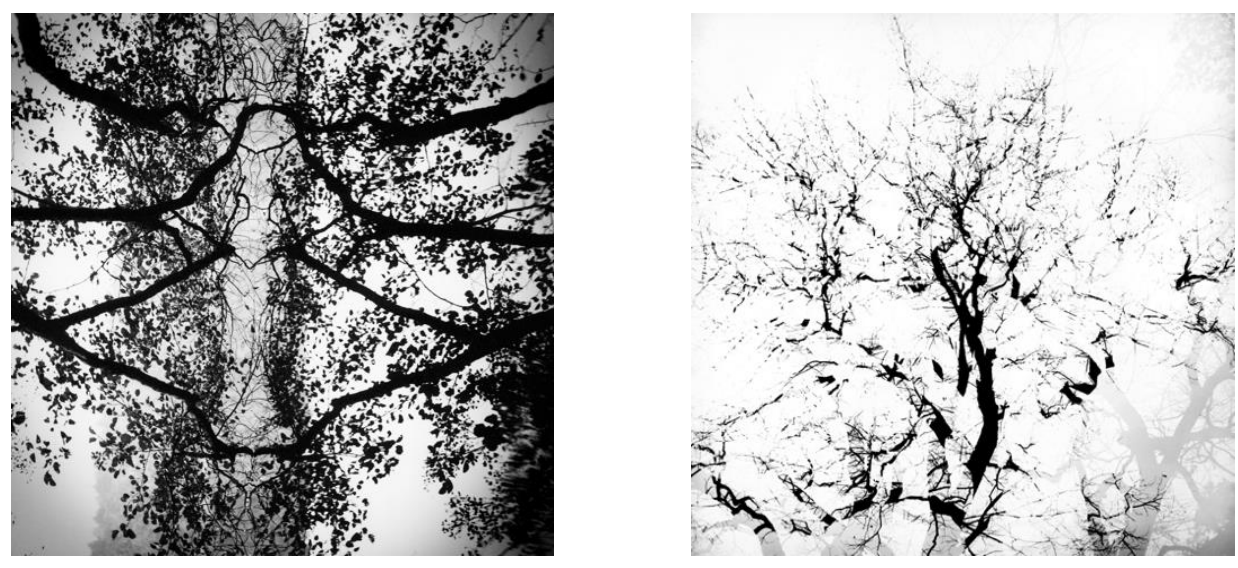
In Caciagli's photos, the encounter between two shapes folds and unfolds nature: the interrupted geometries bend the living being to the vision, the random ramifications explain to the observer an uncertain visibility.

Thus, filtering through the leaves, between the branches, the trunks, reflecting in the water... the light plays with the regularities and irregularities of nature, iteration of motifs and their diversity.

The artist highlights both but, where the living being is at the origin of these plays of light, the diversity of the details of the iterated forms is omnipresent in the photo.

Iterated morphogenesis, hence evocation of fractal forms. Fractals are in fact a beautiful mathematical invention, based on the iteration of a genesis of forms: they express an invariance of scale, a symmetry, that is they describe shapes that are identical to any enlargement of the image.

The iteration of an algorithm, a set of instructions on how to draw a curve - often given as a recursive equation - allows for the generation of remarkable ones.

Here is a very simple one, the Koch fractal (the reader can reconstruct the generating algorithm) and two others whose description, more complex, requires however only one equation.
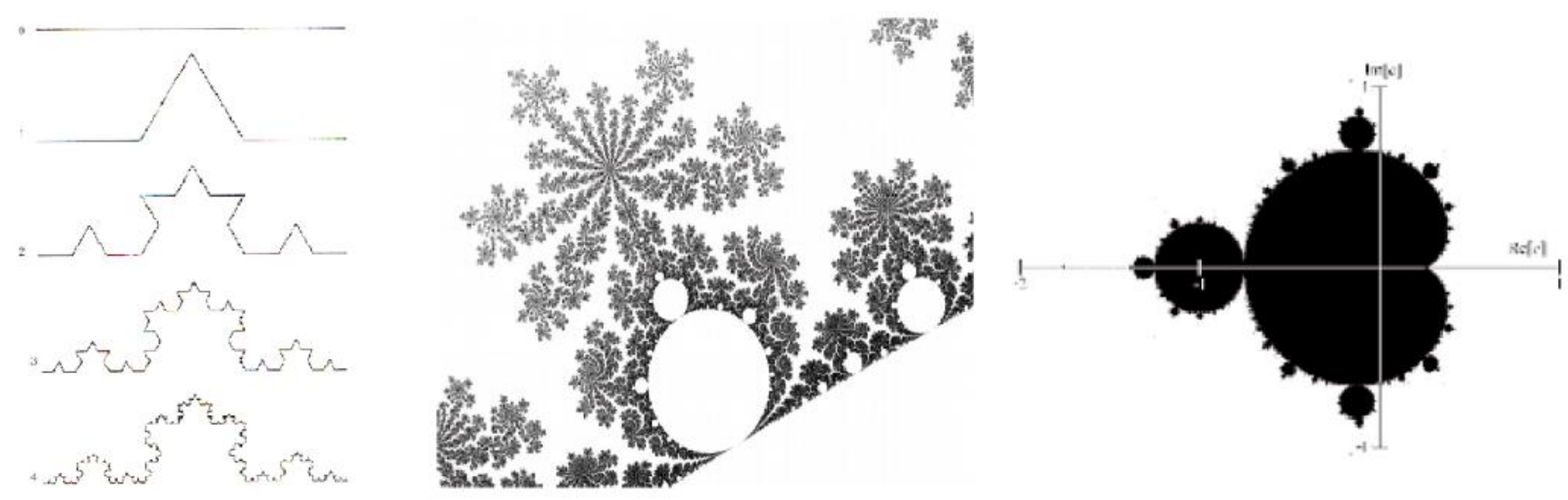

Do they evoke natural forms? Of course, wherever an "iteration of a morphogenetic process" occurs, a fractal can be glimpsed: "self-similarity" characterizes them.

In mathematical physics, more and more of them are proposed in order to understand and to describe: the inert, if subjected to regular play of forces in homogeneous environment, gives rise to diffusion processes that are iterated, self-similar. It is not exactly so in case of an image that refers to the result of a process of living growth. And the artist shows us: fractals not so much fractals, diversity in the iteration. Here is the richness that creates art in Luca Caciagli's photos.

So, let's see why art photography brings us closer to Biology than the current modelling in homogeneous mathematical spaces, including fractals, and this unlike Physics: the living is the result of a "never identical iteration of a morphogenetic process".

In Biology, the reproduction from a cell that splits to a reproducing organism, always occurs with variation: this is the first principle of Darwin's heredity ("Descent with Modification") expressed and insistently iterated, in four of the first six chapters of the Origin of Species: in a sense, each reproduction forces a "symmetry breaking". Modifications are induced, Darwin says, by the "high sensitivity" (!) of the organisms both to internal and external changes - in dynamically heterogeneous ecosystems, we may specify. 
Moreover, mathematical and physical fractals can be "beautiful", the eye is impressed with, but they are not "art". There is not that play of broken symmetries and irregularities that the artist's interpretation proposes, that suggests and claims a glance, also from the viewer.

Caciagli's photos show the result of dynamics of the living being, iterating forms, of course, but always in a different way, well beyond the obsessive and artificial invariance of scale given by an algorithm, produced by a machine.

The artist proposes a point of view, he interprets nature, he looks at it and makes us looking at the surprises that the living being reserves. He therefore underlines the diversity production, in the iteration of forms too, in the reproduction of cells and complex organisms. Caciagli uses further technical tool to diversify the views on nature, to interpret it, to interpret iteration: the double exposure.
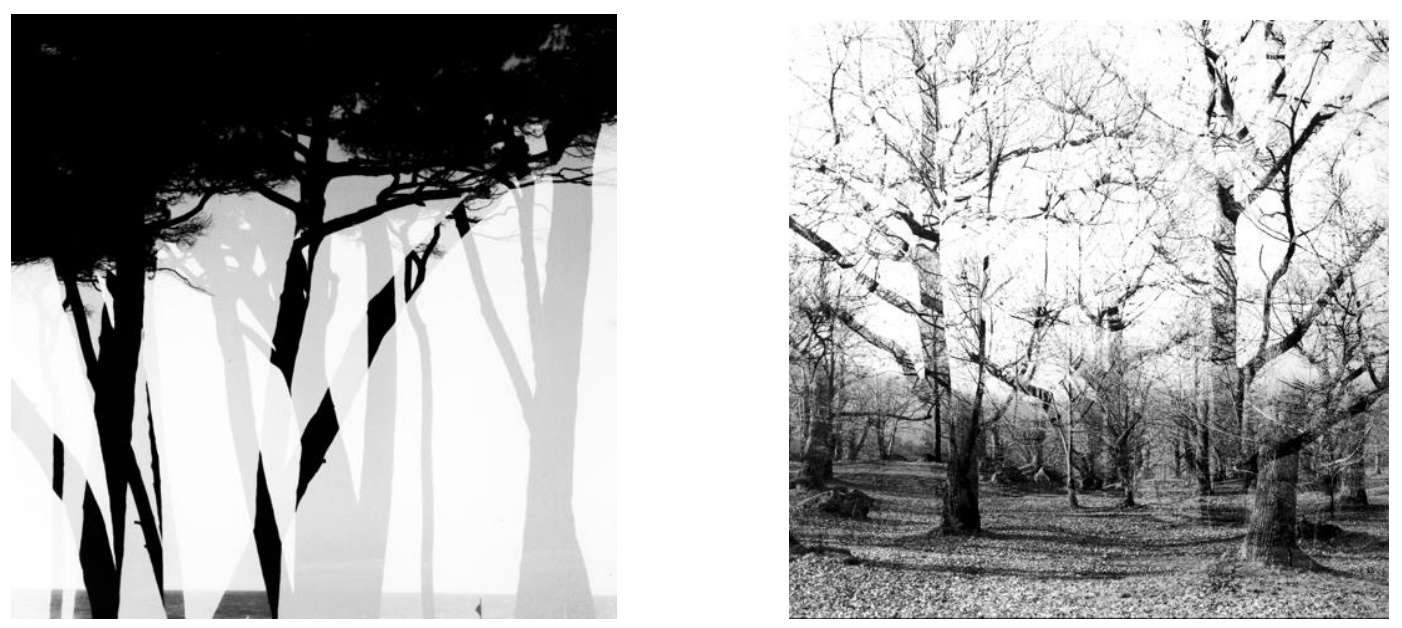

So, branches in front of leaves becoming water fronting trunks...in the photos the gaze walks along the surface following the playing of whites and blacks; the eye is often attracted and stopped by a darker part which is not a shadow but a double layer of matter.

What's foreground and what's behind? Is the darkest branch foreground?

So it would seem but looking more carefully we realize that sometimes it's a light, almost invisible branch which, once overlaid on the dark branch, cancels the latter.

Thanks to the double exposure technique chosen by the artist, one photo meets the other and their matching creates an opacity, giving the graphic embroidery a third dimension.

The thickness turns into bidimensional surface again when the two photos get close, producing white color.

One tree cancels the other, a piece of sky hides a branch and overpoweringly brings back the opaque white of the printing sheet.

This recalls us Magritte's painting, where the tree and the background of the painting switch before the horse, trotting in front of the tree and the background. Not by chance Magritte entitled his painting Le blanc-seing, the vision of white, of the background, of the flat, the sign that the "behind" is "front" at the same time since after all it is on a flat sheet that everything takes place. 


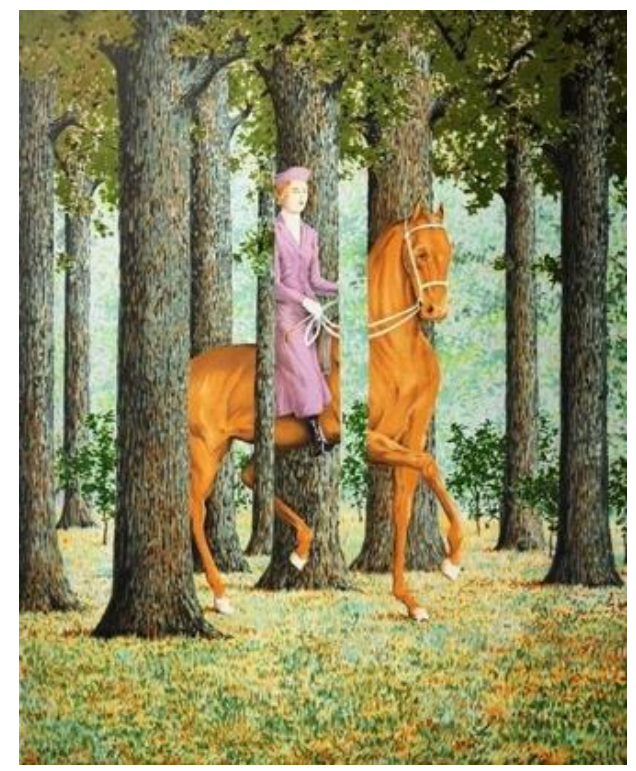

René Magritte, Le blanc-seing, 81,3 × 65,1, oil on canvas, 1965. Washington, National Gallery of Art.

In those shades of white of Caciagli's photos, the composition creates a crease of matter.

Simon Hantaï does it with the canvas itself: he crumples the cloth, dips it in the painting, then stretches and spreads it. Each white spot has the same thickness as the colored spots.

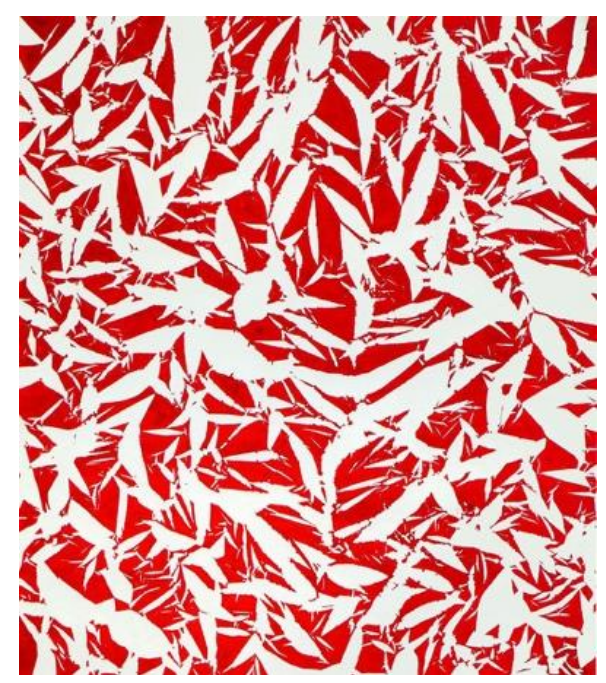

Simon Hantaï, Étude, 1969, oil on canvas, 275 x 238 cm. Washington, National Gallery of Art.

Let's go back to discuss the physical-mathematical significance of fractals and their modest biological relevance, and then talk about the distortion they induce in the analysis of some human dynamics: It is precisely the detachment from the algorithmic abstractness, which may be useful in the analysis of the inert, that allows the photographer's gaze on life of making art.

Classical physics is based on mathematics of the continuum, more precisely on trajectories and smooth (differentiable) surfaces: the measurement, always an interval (it is approximated) does not allow you to go below a threshold where the differentiable continuum no longer makes sense, the threshold of quanta.

Thus, very interesting proposals describe quantum dynamics in terms of fractals (coherent states, condensation of bosons ...), allowing us also to conceive an infinity of optimal paths between two points. We are not talking about the "real", inaccessible in microphysics, but of the effective mathematical descriptions of the continuous and discrete overlapping, typical of the quantum. 
But ... the lungs and the vascular system are they fractals, as many claim? Is it so for any "natural tree" diffusing matter for life by the formation of iterated branching?

Vaguely so ... lungs and arteries are not made of inert, soft matter, fully shaped by physical forces, such as the pre-natal pressure of the inhaled amniotic fluid, of the blood pumped from the embryonic heart, a forced flow. They are instead made of cells that reproduce with variation (Darwin) and move: those physical forces, pressures and flows, act as physical constraints that canalize cells' reproduction with variation and motility in a "roughly" fractal form. Diversity, within a lung and of lungs in a population, contributes to their stability, to "fitness": this is unimaginable if these organs were exact, scale invariant fractals. Moreover, in some cases, such as the mammary glands, no internal flow, no lactation forces their embryogenesis: their vaguely fractal structure (more an iterated disconnected graph) derives from active cell reproduction and motility under the constraints of the tissue texture that they simultaneously generate (see the work by M. Montévil linked below). At lactation, the dispersed branching becomes connected and functional, by its penetrating diversity and irregularities.

Have a look to a field of true sunflowers or cauliflowers, often evoked for the "fractality" of some of their forms: they are all different and each individual violates the alleged fractal structure generated by Fibonacci series, as it is sometimes told. And this diversity, as always in the living, is functional to the resilience of the field, of the single flower, its resistance to changes in the ecosystem. Life dynamics break the symmetries; randomness is not noise, dirt, like in algorithmically created drawings of fractals or in crystals, but variability and diversity production at the core of biological dynamics.

The fractal suggests a background "pattern", not useless to understanding, but highly incomplete. The "causal" analysis must be reversed: first reproduction with variation and motility of cells, then forces constraining the iterated dynamics, thus the quasi-fractal form, the apparent Fibonacci's series.

Caciagli's trees tell us about it very well and the way the artist shows it, by adding to the biological variability the diversity of his sights and of the techniques, does underline it further. Art and science touch each other.

Biology is a science whose time is marked by the production of diversity, by broken symmetries, such as scale invariance / symmetry. So is the economy, yet another historical science. The anhistoricity of physical-mathematical origin makes a caricature of it and often produces disasters. See for example the fractals used in the stock exchange.

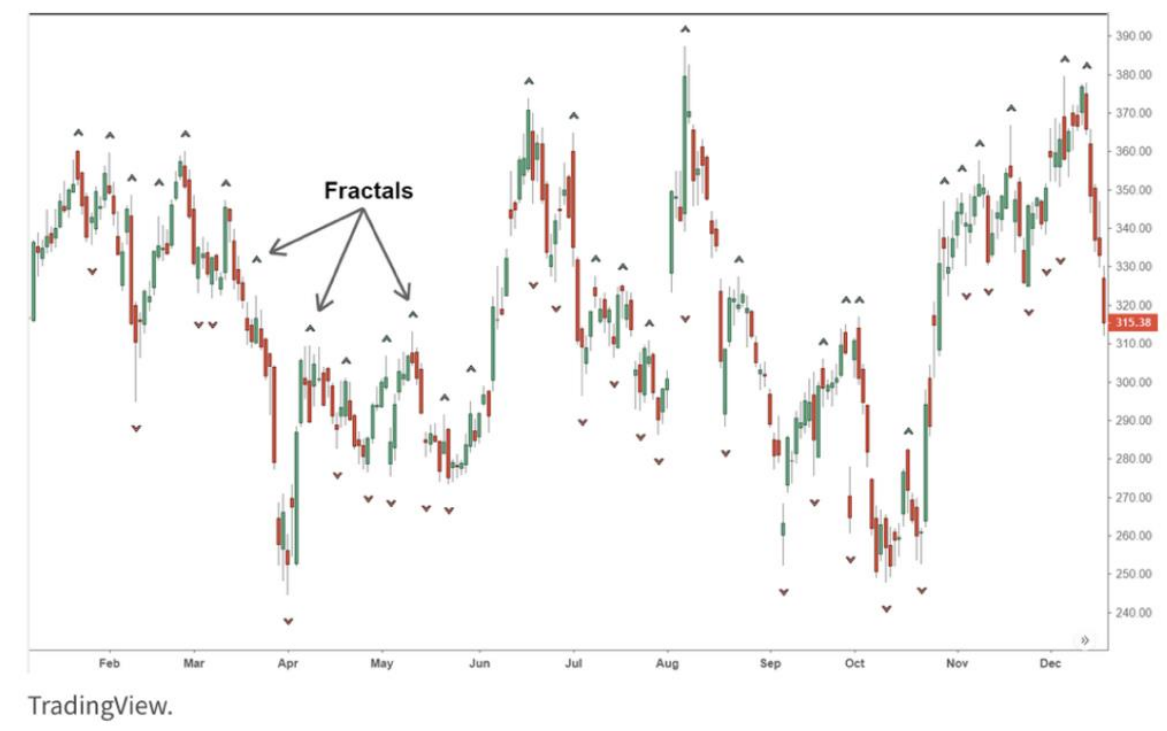


These take part in an analysis of short, very short, ultra-short term trends: often refined mathematical analyses allow to make profits on very rapid price fluctuations, in which those who better identify local regularities, fractals for example, earn more, very far from underlying economic trends.

In other words, non-trivial mathematics helps to hide economic trends: when the profit is mainly or exclusively based on very rapid price fluctuations, we definitely move away from the economic and ecosystem value of investments, not to mention labor-value or production, presumed "underlying values". Nano-second fast-trading helps to make people forget the alleged "priceindicator", the basic trends that may also be destroying the ecosystem.

We need a reconstruction of meaning, in relation to nature, to economy, and the look of art on nature, on its diversity, on the plays of light and possible readings, can make an important contribution to both beauty and knowledge.

We also need to invent, and some of us try, new non-homogeneous mathematics, not only based on symmetries and invariances, but also on their breakings, on the heterogenesis of forms, where randomness is not noise but a component of variability and diversity production. The reader may go to https://cardano.visions-des-sciences.eu/en/content/presentation-0, for the work by A. Sarti, M. Montévil on heterogenesis, its mathematics and biological modeling, as well as, for an analysis of the "fog" diffused by the stock exchange on economy, by N. Bouleau, a mathematician of stochastic processes. These scientific analyses go beyond formal automatisms and the identical iteration of an algorithm, from which also Caciagli's photos take us away with great inventiveness. 